\title{
Genome-wide characterization of SPL gene family in Codonopsis pilosula reveals the novel roles of CPSPL2 and CPSPL10 in promoting the accumulation of secondary metabolites and growth of $C$. pilosula hairy root
}

Jing Yang

Shaanxi Normal University

Zhonglong Guo

Peking University

Yao Cao

Shaanxi Normal University

Rui Chen

Shaanxi Normal University

Wentao Wang

Shaanxi Normal University

Xiaozeng Yang

Beijing Academy of Agriculture and Forestry Sciences

xiaoyan cao ( $\nabla$ caoxiaoyan@snnu.edu.cn )

Shaanxi Normal University https://orcid.org/0000-0002-3188-6663

Research article

Keywords: Biomass, Codonopsis pilosula, Expression patterns, Hairy root, SPLs, Secondary metabolites

Posted Date: November 24th, 2020

DOI: https://doi.org/10.21203/rs.3.rs-81496/v1

License: (1) This work is licensed under a Creative Commons Attribution 4.0 International License. Read Full License 


\section{Abstract \\ Background}

SQUAMOSA PROMOTER BINDING PROTEIN-LIKE (SPL) transcription factors play critical roles in regulating diverse aspects of plant growth and development, including vegetative phase change, plant architecture, anthocyanin accumulation, lateral root growth, etc. Codonopsis pilosula is a famous medicinal plant and its dried root, named Dangshen, is one of the most widely used traditional Chinese medicine. However, little information about SPL genes in this species has been reported.

\section{Results}

In the present study, 15 SPL genes were identified based on the genome data of Codonopsis pilosula. Ten of the 15 CpSPLs were predicted to be the targets of miR156. Phylogenetic analysis clustered CpSPLs into seven groups (G1-G7) along with 16 SPLs from Arabidopsis thaliana. CpSPLs in the same group share similar gene structure and conserved motif composition. Cisacting elements responding to light, stress, and phytohormone widely exist in their promoter regions. Our qRT-PCR results indicated that 15 CPSPLs were differentially expressed in different tissues (root, stem, leaf, flower, and calyx), different developmental periods (1, 2 and 3 months after germination), and various conditions ( $\mathrm{NaCl}, \mathrm{MeJA}$ and ABA treatment). Compared with the control, overexpression of $C p S P L 2$ or $C p S P L 10$ significantly promoted not only the growth of hairy roots, but also the accumulation of total saponins and lobetyolin.

\section{Conclusions}

The SPL genes in the $C$. pilosula genome were identified and their expression patterns were analyzed. The novel roles of CPSPL2 and CPSPL10 in promoting the accumulation of secondary metabolites and growth of $C$. pilosula hairy root were revealed. Our results established a foundation for further investigation of $C P S P L$ s and provided novel insights into their biological functions.

\section{Background}

Transcription factors (TFs) function in various physiological and developmental processes via activating and/or repressing transcription of multiple target genes [1]. They have been usually divided into different families according to the sequence of DNA-binding domains and other conserved motifs [2]. SQUAMOSA-promoter binding protein-like (SPL or SBP) TFs are exclusive to plant and characterized by a highly conserved SBP domain and a nuclear localization signal (NLS) at the C-terminus. The SBP domain is approximately 76 amino acids and includes two zinc-binding sites (one zinc finger is $\mathrm{C} 3 \mathrm{H}$ or $\mathrm{C} 4$, and the other is $\mathrm{C} 2 \mathrm{H} 4$ ) essential for DNA binding, and the NLS partially overlapps with the second zinc finger [3-6]. AmSBP1 and AmSBP2 from Antirrhinum majus were the first discovered SBP-domain proteins in plants, and were found to bind to the floral meristem identity gene SQUAMOSA promoter, so named them [3]. Then SPL genes have been identified in many plant species, including single-cell algae, mosses, gymnosperms, and angiosperms [7]. With the rapid implication of high-throughput sequencing technology, more and more plant genome data have been released and genome-wide identification of the $S P L$ gene family from model and non-model plants have been identified in Arabidopsis thaliana [8], Oryza sativa [9], Glycine max [10], Solanum lycopersicum [11], Malus domestica [12], Salvia miltiorrhiza [13], Vitis vinifera [14], Phyllostachys edulis [15], Capsicum annuum [16], Ricinus communis [17], etc.

The functions of SPL genes have been well characterized in the model plant Arabidopsis and they play important regulatory roles in diverse developmental progresses, including vegetative to reproductive phase transition, cotyledon- to vegetative-leaf transition, micro- and megasporogenesis, trichome formation, stamen filament elongation, axillary bud formation, and lateral root growth [18-23]. Besides, they are involved in copper homeostasis, abiotic stress response, immune response, and secondary metabolites production [24-27]. The functions of $S P L$ genes from other species have also been identified. In rice, OSSPL 14 has been found to promote panicle branching and grain productivity and OSSPL16 regulates grain yield and quality

Page $2 / 20$ 
$[28,29]$. FVSPL 10 from strawberry (Fragaria vesca) not only promotes early flowering, but also increases organs size, such as longer root, larger floral organ and seeds [30]. As a class of plant-specific gene family, some SPL genes are important candidates for improving plant agronomic traits by genetic engineering.

Codonopsis pilosula is a member of the Campanulaceae family. Its dried root, named "Dangshen" in Chinese, is one of the most widely used traditional Chinese medicine for replenishing qi (vital energy), strengthening body immunity, improving appetite, promoting gastrointestinal function, reducing blood pressure, and curing gastric ulcers [31]. In addition, Dangshen is also a wellknown health-care food in China and is listed in the "Food and Drug Homology Catalogue" approved by the National Health Commission of People's Republic of China. Consequently, the demand for Dangshen is growing, and the yield and accumulation of bioactive metabolites is attracting more and more attention in planting field [32, 33]. Lobetyolin, alkaloids, polysaccharides, and saponins are the major active ingredients in Dangshen, which are responsible for most of the pharmacological functions found in the medicine [34]. Lobetyolin, a general marker compound in Dangshen, has been well reported to exert multiple bioactivities, such as anti-cancer, antiviral, anti-inflammatory, anti-oxidative, mucosal protective, and xanthine oxidase inhibiting properties $[35,36]$.

Although $C$. pilosula has received great attention on the chemical constituents and their pharmacological activities, relevant study of this species at the genetic level is lagging behind and only a few literatures involved genes in C. pilosula [37-40]. Until now, SPL gene family has never been reported in C. pilosula. Most recently, we have developed an efficient Agrobacterium rhizogenes-mediated transformation approach for transgenic hairy roots with this species [39], which lay a good foundation for genetic engineering of that species. Here, we identified 15 SPL genes based on the genome sequence of $C$. pilosula (data

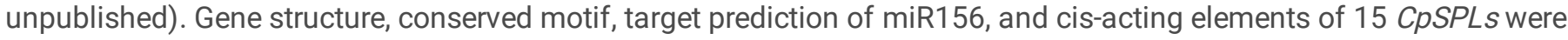
systematically analyzed. And their spatiotemporal expression profiles in different tissues and expression patterns under various conditions ( $\mathrm{NaCl}$, MeJA and ABA treatment) were analyzed by qRT-PCR. Furthermore, we obtained CpSPL2 or CpSPL 10 overexpressing transgenic hairy roots, and a significant increase was observed in the biomass and concentrations of total saponins and lobetyolin. As far as we know, this is the first experimental research on gene function in this species. These findings demonstrate that $C P S P L 2$ and $C P S P L 10$ positively regulate the growth of hairy roots and accumulation of active ingredients, which have great potential in improving the yield and quality of Dangshen.

\section{Methods}

\section{Identification of SPL genes in C. pilosula and bioinformatic analysis}

The sequences of SBP domain (ID: PF03110), which were downloaded from Pfam database (http://pfam.xfam.org/), was used to search possible SPL genes in C. pilosula genome sequences (data unpublished) by HMMER (http://hmmer.org) with the evalue $<1 \mathrm{e}-10$. A total of $15 \mathrm{CPSPL}$ genes containing a complete SBP domain were identified.

The online analysis software psRNATarget (http://plantgrn.noble.org/psRNATarget/analysis?function=3) was used to predict CPSPL genes directly targeted by miR156, with the maximum expectation value of 3.0 and UPE value of 16. MEGA X software (https://mega.nz/) was used to construct the phylogenetic tree of 31 full-length SPL amino acid sequences, 15 from $C$. pilosula and 16 from $A$. thaliana, with 1000 bootstraps in the Maximum-likelihood (ML) method. Gene Structure Display Server (http://gsds.cbi.pku.edu.cn/) was used for gene structure analysis. The MEME program (http://meme-suite.org/) was used to for identification of the conserved motifs. The cis-acting elements of 15 CpSPL genes promoter regions (2000 bp upstream of the translation initiation codon "ATG") were analyzed online (http://bioinformatics.psb.ugent.be/webtools/plantcare/html/).

\section{Plant materials and treatments}

Seeds of $C$. pilosula were collected from Gansu Province, China. The botanical origin of the materials was identified by Professor ZheZhi Wang in Shaanxi Normal University. The specimens of the seeds were deposited in the herbarium of National Engineering Laboratory for Resource Development of Endangered Crude Drugs in Northwest of China, Shaanxi Normal University, Xi'an, China. The Seed owner allowed the collection. The seeds of $C$. pilosula were germinated and incubated according to the method that we described previously [39].

Page $3 / 20$ 
For gene spatiotemporal expression analysis, the leaves, stems, and roots were collected separately from one-, two-, and threemonth-old seedlings, and the flower and calyx were collected from the plants at the flowering stage. To test CPSPLs responses to hormonal and stress treatments, two-week-old seedings were treated with $200 \mathrm{mmol} \mathrm{NaCl}, 200 \mu \mathrm{mol}$ MeJA, and $100 \mu \mathrm{mol}$ ABA, respectively, as we have described previously [40]. The control group was treated with the same amount of ddd $\mathrm{H}_{2} \mathrm{O}$ and all the samples were collected $6 \mathrm{~h}$ after treatment.

\section{Gene expression analysis}

For qRT-PCR analysis, total RNA was extracted and then reverse transcripted into cDNA as we described previously [40]. All the primer sequences used for qRT-PCR were listed in Table S1 and $C p G A P D H$ was used as the internal control [40]. The relative expression levels of 15 CPSPLs were calculated according to the method described by Livak and Schmittgen (2001) [41]. All the experiments included three biological and three technical replicates.

\section{Vector construction and hairy root transformation}

The complete open reading frames of $C P S P L 2$ and $C P S P L 10$ were amplified through PCR using the specific primer pairs CpSPL2-F/R and CpSPL 10-F/R (Table S1), respectively, with the following PCR conditions: $98{ }^{\circ} \mathrm{C}, 3 \mathrm{~min} ; 30$ cycles of $98{ }^{\circ} \mathrm{C}, 10 \mathrm{~s}$, $58{ }^{\circ} \mathrm{C}, 30 \mathrm{~s}, 72{ }^{\circ} \mathrm{C}, 45 \mathrm{~s} ; 72{ }^{\circ} \mathrm{C}, 5 \mathrm{~min}$. Then the products were digested with $\mathrm{Pacl}$ and $A s c \mathrm{l}$ and ligated into pMDC85 to generate overexpression (OE) vectors pMDC85-CPSPL2 and pMDC85-CPSPL 10.

Transgenic hairy roots overexpressing $C P S P L 2$ or $C P S P L 10$ were obtained by Agrobacterium-mediated method according to the protocol established in our lab and selected on the MS medium containing $2 \mathrm{mg} / \mathrm{L}$ hygromycin [39]. In parallel, pMDC85 was introduced into $C$. pilosula as the empty vector control (EV). Every transgenic line was excised and sub-cultured separately as we described previously [39]. Five independent $C P S P L 2-O E$ lines, seven $C P S P L 10-0 E$ lines, and four EV lines were obtained, and then confirmed by genomic DNA PCR using primers hptI/F/R (Table S1) for hygromycin phosphotransferase II gene (hptII), followed by expression analysis of CPSPL2 or CPSPL 10 by qRT-PCR.

\section{Determination of lobetyolin and total saponins}

Transgenic hairy roots sub-cultured for one month were used for determination of lobetyolin and total saponins.

To determine the concentration of lobetyolin, we ground the dried hairy roots into powder, followed by extracted three times with $10 \mathrm{~mL}$ methanol by sonication (50-150 W) in an ultrasonic bath (Kunshan Instrument Co., Ltd., China) for 30 min, 20 min, and $15 \mathrm{~min}$, respectively. The extracts were put together and the methanol solution was evaporated, followed by dissolved with methanol to $5 \mathrm{~mL}$ volumetric flask. After filtration with $0.22 \mu \mathrm{m}$ microporous membrane, the solution was used for HPLC analysis on a Shimadzu LC-20A instrument (Shimadzu, Japan) equipped with an Agilent 5 TC-C 18 column $(250 \mathrm{~mm} \times 4.6 \mathrm{~mm}$,

$5 \mu \mathrm{m})$. The mobile phase consisted of ultrapure water (A) and methanol (B) and the gradient condition was $0-5 \mathrm{~min}, 20-40 \% \mathrm{~B}$; 5-10 min, 40-70\% B; 10-12 min, 79-90\% B; 12-25 min, 90\% B. The separation was performed at $30{ }^{\circ} \mathrm{C}$, with the flow rate of $1.0 \mathrm{~mL} / \mathrm{min}$ and UV detector wavelength at $220 \mathrm{~nm}$.

The concentration of total saponins in transgenic hairy roots was determined as we described previously [39].

\section{Statistical analysis}

All the experiments and date presented here involved at least three biological repeats. SPSS version 20.0 software (SPSS Inc., Chicago, IL, USA) was used for statistical evaluation. The error bars indicate standard deviation. Significant difference of the mean values was set at $P<0.05$.

\section{Results}

\section{Genome-wide identification and sequence feature analysis of CpSPLs}

To identify possible SPL genes in C. pilosula genome sequences, we employed the SBP domain (PF03110) to search the databases by HMMER. A total of 15 SPLs containing complete SBP domain were identified based on the genome sequence of 
C. pilosula and their cDNA sequences were listed in (Table S2). Consulting the homologous AtSPLs in Arabidopsis, 15 CpSPLs were named from $C P S P L 1$ to $C P S P L 15$. The deduced CpSPLs exhibited great variations in terms of their molecular weight (MW), ranging from $17.98 \mathrm{KDa}(C P S P L 5)$ to $119.80 \mathrm{KDa}$ (CPSPL 14). Similarly, the lengths of the CDS were found to be varied in the CpSPLs, from $480 \mathrm{bp}$ (CPSPL5) to $3276 \mathrm{bp}$ (CPSPL 14). The detailed information, including the gene length, intron number, protein length, predicted MW, and theoretical isoelectric point (pl), were listed in Table 1.

Table 1

The information of 15 SPL genes in Codonopsis pilosula

\begin{tabular}{|c|c|c|c|c|c|c|c|c|c|}
\hline $\begin{array}{l}\text { Gene } \\
\text { name }\end{array}$ & $\begin{array}{l}\text { No. } \\
\text { intron }\end{array}$ & $\begin{array}{l}\text { Gene } \\
\text { length } \\
\text { (bp) }\end{array}$ & $\begin{array}{l}\text { CDS } \\
\text { length } \\
\text { (bp) }\end{array}$ & $\begin{array}{l}\text { Protein } \\
\text { siza(aa) }\end{array}$ & $\mathrm{Mw}(\mathrm{Da})$ & pl & Atomic composition & GRAVY & $\begin{array}{l}\text { Instability } \\
\text { Index }\end{array}$ \\
\hline CpSPL 1 & 10 & 10066 & 3036 & 1011 & 112293.93 & 7.10 & $\mathrm{C}_{4918} \mathrm{H}_{7801} \mathrm{~N}_{1421} \mathrm{O}_{1515} \mathrm{~S}_{38}$ & -0.395 & 54.03 \\
\hline CPSPL2 & 4 & 3264 & 879 & 292 & 32767.81 & 8.74 & $\mathrm{C}_{1414} \mathrm{H}_{2225} \mathrm{~N}_{415} \mathrm{O}_{447} \mathrm{~S}_{18}$ & -0.534 & 67.85 \\
\hline CPSPL3 & 2 & 6640 & 1095 & 364 & 40962.56 & 8.70 & $\mathrm{C}_{1778} \mathrm{H}_{2735} \mathrm{~N}_{545} \mathrm{O}_{544} \mathrm{~S}_{16}$ & -0.708 & 58.47 \\
\hline CpSPL4 & 1 & 3004 & 618 & 205 & 23029.53 & 6.20 & $\mathrm{C}_{964} \mathrm{H}_{1560} \mathrm{~N}_{310} \mathrm{O}_{324} \mathrm{~S}_{11}$ & -0.993 & 68.72 \\
\hline CPSPL5 & 1 & 4163 & 480 & 159 & 17978.16 & 9.26 & $\mathrm{C}_{753} \mathrm{H}_{1218} \mathrm{~N}_{252} \mathrm{O}_{239} \mathrm{~S}_{11}$ & -1.005 & 44.17 \\
\hline CpSPL6 & 3 & 6537 & 1602 & 533 & 58148.49 & 8.55 & $\mathrm{C}_{2492} \mathrm{H}_{3928} \mathrm{~N}_{746} \mathrm{O}_{817} \mathrm{~S}_{23}$ & -0.662 & 47.64 \\
\hline CpSPL7 & 10 & 13540 & 2391 & 796 & 89393.21 & 6.52 & $\mathrm{C}_{3932} \mathrm{H}_{6209} \mathrm{~N}_{1099} \mathrm{O}_{1182} \mathrm{~S}_{50}$ & -0.36 & 58.97 \\
\hline CPSPL8 & 3 & 2322 & 957 & 318 & 35386.95 & 8.40 & $\mathrm{C}_{1531} \mathrm{H}_{2332} \mathrm{~N}_{454} \mathrm{O}_{490} \mathrm{~S}_{14}$ & -0.777 & 59.08 \\
\hline CpSPL9 & 2 & 19029 & 1140 & 379 & 40795.13 & 8.38 & $\mathrm{C}_{1767} \mathrm{H}_{2718} \mathrm{~N}_{530} \mathrm{O}_{558} \mathrm{~S}_{15}$ & -0.713 & 63.79 \\
\hline CpSPL 10 & 4 & 7056 & 1383 & 460 & 50220.85 & 8.40 & $\mathrm{C}_{2185} \mathrm{H}_{3399} \mathrm{~N}_{631} \mathrm{O}_{698} \mathrm{~S}_{17}$ & -0.577 & 52.76 \\
\hline CpSPL 11 & 4 & 3304 & 1077 & 358 & 40255.95 & 8.69 & $\mathrm{C}_{1731} \mathrm{H}_{2717} \mathrm{~N}_{535} \mathrm{O}_{541} \mathrm{~S}_{18}$ & -0.717 & 54.19 \\
\hline CpSPL 12 & 3 & 3095 & 969 & 322 & 35688.83 & 9.11 & $\mathrm{C}_{1524} \mathrm{H}_{2415} \mathrm{~N}_{463} \mathrm{O}_{493} \mathrm{~S}_{18}$ & -0.641 & 60.11 \\
\hline CpSPL 13 & 2 & 3364 & 1176 & 391 & 43540.24 & 6.52 & $\mathrm{C}_{1874} \mathrm{H}_{2908} \mathrm{~N}_{552} \mathrm{O}_{608} \mathrm{~S}_{20}$ & -0.709 & 61.52 \\
\hline CpSPL 14 & 10 & 6316 & 3276 & 1091 & 119799.88 & 8.47 & $\mathrm{C}_{5200} \mathrm{H}_{8251} \mathrm{~N}_{1549} \mathrm{O}_{1616} \mathrm{~S}_{46}$ & -0.479 & 51.64 \\
\hline CpSPL 15 & 2 & 10641 & 1140 & 379 & 40765.11 & 8.38 & $\mathrm{C}_{1766} \mathrm{H}_{2716} \mathrm{~N}_{530} \mathrm{O}_{557} \mathrm{~S}_{15}$ & -0.712 & 63.05 \\
\hline
\end{tabular}

Prediction of CpSPLs targeted by miR156

miR156, one of the most conserved miRNA families, plays very important roles in the process of plant growth and development by direct cleavage of $S P L$ transcripts [42]. In the model plant Arabidopsis, 10 of 16 AtSPLs are direct targets of AtmiR156 [42]. In rice, 11 of 19 OsSPL genes are targeted by OsmiR156 [9]. We predicted CpSPLs targeted by AtmiR156 using the on line plant

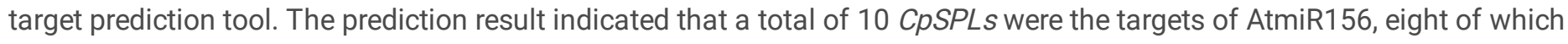
(including CPSPL3, CPSPL6, CPSPL9-13, and CPSPL15) were targeted in the coding regions, while two (CPSPL4 and CPSPL5) in 3' UTR regions (Fig. 1).

\section{Phylogenetic analysis of CpSPLs}

We constructed a phylogenetic tree of 15 CpSPLs and 16 AtSPLs using MEGA X with ML method. As shown in Fig. 2, 31 SPLs from two species were classfied into seven groups, named from $\mathrm{G} 1$ to $\mathrm{G} 7$, and each group consisted of at least one SPL from $C$. pilosula and one from A. thaliana. There are only two members in G3 (AtSPL8 and CPSPL8) and G7 (AtSPL7 and CPSPL7). G5 is the largest group with three AtSPLs (AtSPL6/9/15) and four CPSPLs (CPSPL3/6/9/15). It was interesting that all the members in 
G1, G3, and G7 belong to non-miR156-targeted SPLs, including six AtSPLs (AtSPL 1/ 7/ 8/12/ 14/ 16) and four CpSPLs (CpSPL1/7/8/ 14). Except for CPSPL2, All the members in G2, G4, G5, and G6 were miR156-targeted SPLs. In Arabidopsis, AtSPL2/ 10/11, three members closely related, regulate root regeneration by inhibiting auxin biosynthesis [43]. Phylogenetic tree clustered CPSPL2, CPSPL 10, AtSPL2, AtSPL10, and AtSPL 11 in G4, indicating CpSPL2 and CpSPL 10 are probably involved in root growth.

\section{Gene structure and conserved motif analysis}

To clarify the structural diversities of 15 CPSPLs, we performed gene exon/intron structure analysis. The result displayed that the number of introns had a high variation and ranged from one to ten (Fig. 3). Interestingly, we found that most CpSPLs in the same group share similar structure. For instance, $C P S P L 1$ and $C P S P L 14$, belonging to $\mathrm{G} 1$, have ten introns, respectively. $C p S P L 4$ and $C P S P L 5$, members in G6, have only one intron, respectively (Fig. 3).

To explore the conserved motifs, 15 CPSPLs were subjected to analysis with MEME program. Among the 12 conserved motifs identified (Fig. 4, Table S3), motif 1, motif 2, and motif 3 existed in all the 15 CpSPLs and formed the conserved SBP domain. Similar motif composition existed in the same group. For example, CPSPL2 and CPSPL10 in G4 all consisted of five conserved motifs (motif 1/2/3/10/11). The motif composition in CPSPL 9 was completely consistent with that in $C P S P L 15$, suggesting that CPSPL 9 and $C P S P L 15$ probably have similar and redundant functions in plant development.

\section{Cis-acting elements analysis of CpSPLs promoter regions}

We analyzed the cis-acting elements of 15 CpSPLs promoter regions and light responsive elements (including G-box, GATAmotif, GTGGC-motif, AE-box, TCT-motif, and chs-CMA2a), hormone responsive elements [such as gibberellin (GARE-motif), MeJA (CGTCA- and TGACG-motif), and abscisic acid (ABA) (ABRE)], stress responsive elements [such as drought (MBS), lowtemperature (LTR), and anaerobic induction (ARE)], and CAT box related to meristem expression were found in their promoter regions (Table S4). Among these cis-elements, MeJA-responsive elements existed in the promoter regions of almost all the CPSPLs except for CPSPL9 and CPSPL 13, and ABA-responsive element (ABRE) existed in the promoter regions of 10 CpSPLs (including CPSPL 1, CPSPL6-10, CPSPL 12, and CPSPL14-15).

\section{Spatiotemporal expression analysis of CPSPL genes}

We investigated the expression patters of 15 CPSPLs in the leaves, stems, and roots from one-, two-, and three-month-old seedlings, and the flower and calyx from the plants at the flowering stage by qRT-PCR assay. The results showed that most CPSPLs expressed in almost all the tissues (Fig. 5). Compared with other genes, the expression level of CpSPL 7 was more constant in all the tissues tested. CPSPL8 showed highest level in calyx. CPSPL5 was expressed at relatively higher levels in leaf and calyx. The expression levels of CPSPL3, CPSPL8, CPSPL10, CPSPL12, and CPSPL 13 in the stems gradually decreased with the maturation of the seedlings. $C P S P L 1$ and $C P S P L 14$, two members in $\mathrm{G} 1$, showed similar expression patterns and their expression levels in the root increased gradually with the maturation of the seedlings. In addition, the expression patterns of CPSPL9 and CPSPL 15 were highly similar, with higher levels in flowers and 3-month-old roots. In summary, spatiotemporal expression analysis results indicated that $C P S P L$ genes exhibited various expression patterns, which provide preliminary information for understanding their potential functions in the development of $C$. pilosula.

\section{Expression profiles of CpSPLs under various conditions}

To assess the expression profiles of $15 \mathrm{CpSPL}$ genes under various treatments ( $\mathrm{NaCl}, \mathrm{MeJA}$, and $\mathrm{ABA}$ ), a histogram was generated using the relative expression level (Fig. 6). When treated with $\mathrm{NaCl}$, the transcript levels of eight $\operatorname{CpSPLS}$ (CPSPL 1 , CpSPL2, CpSPL4, CpSPL6, CpSPL7, CpSPL 11, CpSPL 14 and CpSPL15) and four CpSPLs (CpSPL5, CpSPL8, CpSPL 10, and $C P S P L 13$ ) were significantly up-regulated and down-regulated, respectively. Among those, $C P S P L 2, C P S P L 6$, and $C P S P L 11$ increased to 6.02, 5.66, and 7.94 times than the control, respectively, while CPSPL5 and CPSPL8 decreased to 20.00 and 12.50 times than the control, respectively (Fig. 6A). For MeJA treatment, the transcript levels of CpSPL4, CpSPL6, CpSPL 14 and CPSPL15 significantly increased, with the highest change folds in CPSPL15 (3.03 folds). The transcript levels of five CPSPL 
genes (CPSPL3, CPSPL5, CPSPL8, CpSPL10, and CPSPL11) significantly decreased, with 14.28, and 10.00 change folds in CPSPL5 and CPSPL8, respectively (Fig. 6B). Under ABA treatment, eight CpSPLs (CpSPL1, CpSPL4, CpSPL6, CpSPL7, CpSPL9, CPSPL12, CPSPL 14 and CPSPL15) responded positively to the treatment, while three genes (CPSPL3, CpSPL5 and CPSPL8) responded negatively to $A B A$ treatment. Among those genes, $C P S P L 15$ and $C P S P L 8$ exhibited highest upregulation and downregulation, respectively (Fig. 6C).

\section{Overexpression of CpSPL2 or CpSPL10 promotes the growth of C. pilosula hairy root}

To investigate the function of $C P S P L 2$ and $C P S P L 10$ in root development, we generated $C P S P L 2$-overexpressing or $C P S P L 10-$ overexpressing transgenic hairy roots. The expression level of $C P S P L 2$ or $C P S P L 10$ in the transgenics was examined by qRT-PCR (Fig. 7A, B). Two independent CPSPL2-overexpressing lines (CPSPL2-OE3 and CPSPL2-OE5) and CPSPL 10-overexpressing lines (CPSPL10-0E2 and CPSPL2-OE3) with dramatically elevated CPSPL2 or CPSPL 10 expression were selected for further analysis. In comparison to the control, the hairy roots overexpressing CPSPL2 or CPSPL 10 grew faster (Fig. 7C, D, and E). When the transgenic hairy roots with the length about $1.0 \mathrm{~cm}$ were cultured for one month, the biomass of CpSPL2-OE3, CPSPL2-OE5, CPSPL10-OE2, and CPSPL10-OE3 was 2.19, 1.98, 3.15, and 2.83 times that of the control (EV2), respectively (Fig. 7F). Our results indicated that both $C P S P L 2$ and $C P S P L 10$ promote the growth of hairy roots.

\section{Overexpression of CpSPL2 or CpSPL10 promotes accumulation of lobetyolin and total saponins in C. pilosula hairy root}

To evaluate the impact of $C P S P L 2$ or CPSPL 10 on active ingredients, HPLC and UV spectrophotometer were used to determine the concentrations of lobetyolin and total saponins in those transgenic lines, respectively. It was surprising that the production of both lobetyolin and total saponins were greatly increased in CPSPL2-0E or CPSPL 10-0E lines. The concentration of lobetyolin in CPSPL2-OE3, CPSPL2-OE5, CPSPL10-OE2, and CPSPL10-OE3 was 6.43, 6.25, 6.29, and 7.03 times that of the control (EV2), respectively (Fig. 8A). The concentration of total saponins in CPSPL2-OE3, CPSPL2-0E5, CPSPL10-0E2, and CPSPL 10-0E3 was $3.18,2.72,1.81$, and 1.94 times that of the control (EV2), respectively (Fig. 8B). In summary, CpSPL2 and CpSPL10 promote not only the growth of hairy roots but also accumulations of lobetyolin and total saponins.

\section{Discussion}

Identification of SPL genes in C. pilosula

SPLs are plant-specific TFs and characterized by a highly conserved SBP domain $[5,6]$. They play critical roles in regulating diverse aspects of plant growth and development, including vegetative phase change, plant architecture, anthocyanin accumulation, lateral root growth, etc [18-24]. Since its first discovery in A. majus [3], the SPL gene family from various plants has been isolated and identified. For instance, there are $16 \mathrm{SPL}$ gene family members in Arabidopsis thaliana [8], 19 in Oryza sativa [9], 15 in Solanum lycopersicum [11], 15 in Salvia miltiorrhiza [13], and 15 in Ricinus communis [17]. However, little information is known about SPL gene family in C. pilosula, a famous species with important medical and edible values. Here, we identified 15 CPSPL genes in C. pilosula genome. Since most SPL genes are targets of miR156 [42], we predicted miR156targeted CPSPL genes by psRNATarget. Prediction results showed that ten CPSPL genes were targeted by miR156 (Fig. 1), indicating the miR156-SPL module is universal in plants.

We constructed the phylogenetic tree of 16 AtSPLs from A. thaliana and 15 CPSPLs from C. pilosula. 31 SPL genes were divided into seven groups and each group had at least one CPSPL and one AtSPL (Fig. 2). All the non-miR156-targeted AtSPLs and CpSPLs were grouped into G1, G3, and G7. CpSPL family members in the same group showed similar gene structure and motif composition (Fig. 3; Fig. 4), which was consistent with previous report [17]. In Arabidopsis, members in the same group often have the same or similar function. For instance, AtSPL3, AtSPL4, and AtSPL5, clustered in G6, synergistically induce flowering under long-day photoperiod [44]. Most recently, AtSPL2, AtSPL10, and AtSPL11, members in G4, have been reported to inhibit root regeneration by dampening auxin biosynthesis [43]. We speculate that CpSPLs in the same group maybe have the same function, such as CPSPL2 and CPSPL 10 in G4, CPSPL4 and CPSPL5 in G6 and so on.

CpSPL genes expression patterns in C. pilosula

Page 7/20 
Gene expression patterns, to a large extent, will provide valuable information for its potential function [45]. In this study, the spatiotemporal expression patters of 15 CPSPLs in the leaves, stems, and roots from one-, two-, and three-month-old seedlings, and the flower and calyx from the plants at the flowering stage were detected by qRT-PCR (Fig. 5). The results showed that CPSPL 1 and CPSPL14 in G1 exhibited similar expression patterns, and the expression patterns of paralogous CPSPL9 and CPSPL 15 in G5 showed high similarity. Our results were consistent with previous conclusion that paralogous $S P L$ genes in the same group often showed similar expression profiles [46, 47]. AtSPL9, AtSPL 10, and AtSPL 15 contribute to the vegetative to reproductive phase transition [8]. Here, $C P S P L 9, C P S P L 10$, and $C P S P L 15$ expressed predominantly in the flower, suggesting they might function in the development of flower in C. pilosula. The expression level of non-minR156-targeted CpSPL7 was more constant in all the tissues tested, which was consistent with the result of SmSPL 7 from Salvia miltiorrhiza [13].

Some SPL genes have been proved to be involved in abiotic stress. For example, in Arabidopsis, AtSPL 1 and AtSPL 12 function redundantly in thermotolerance and overexpression of AtSPL 1 or AtSPL 12 increased plant thermotolerance [48]. In alfalfa, silencing MSSPL 13 enhanced tolerance to drought and heat stress $\left(40^{\circ} \mathrm{C}\right)[26,49]$, and down-regulation of MsSPL 8 led to

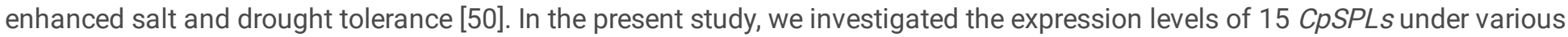
stress conditions, including $\mathrm{NaCl}$, MeJA, or ABA treatment. We found that the expression levels of most $C p S P L$ genes significantly changed under $\mathrm{NaCl}$, MeJA, and ABA treatment (Fig. 6). Among those genes with significant change, $C p S P L 4$, CPSPL6, CPSPL14, and CPSPL 15 positively response to all the treatments, while CPSPL5, CPSPL8, and CpSPL10 negatively response to all the treatments. Compared with other genes, CPSPL5 and CPSPL8 showed higher fold change under different treatments. We speculate that those two genes are potential candidates involved in abiotic stress.

\section{Functional study of the CpSPL2 and CPSPL10 genes}

Since the medicinal and edible part of $C$. pilosula is the root, increasing root yield is one of the main goals of breeding for this species. In Arabidopsis, AtSPL2, AtSPL 10, and AtSPL11, inhibit root regeneration by dampening auxin biosynthesis [43]. The miR156-targeted $S P L 10$ is involved in regulating not only lateral root growth but also primary root growth [21, 23]. Recently, it was reported that overexpression of FVSPL10, a SPL gene from Fragaria vesca, resulted in increased organs size, including longer root, larger floral organ and seeds [30]. We speculated that $C P S P L 2$ and $C p S P L 10$, two members clustered in the same group with AtSPL2/ 10/11 (Fig. 2), were probably involved in the regulation of root development. To investigate the function of CPSPL2 and CPSPL10, we generated transgenic hairy roots overexpressing CPSPL2 or CPSPL 10. Compared with the control, transgenic lines overexpressing CPSPL2 or CPSPL 10 grew faster and the biomass of CPSPL2-0E3, CpSPL2-0E5, CpSPL 10-0E2, and $C P S P L 10-0 E 3$ was 2.19, 1.98, 3.15, and 2.83 times that of the control when the transgenic hairy roots with the length about $1.0 \mathrm{~cm}$ were cultured for one month (Fig. 7). Our results indicated that overexpression of $C P S P L 2$ or CPSPL 10 significantly promote the growth of hairy root.

Furthermore, we determined the concentration of lobetyolin and total saponins in those transgenic lines. Unexpectedly, we found that overexpressing CPSPL2 or CPSPL10 dramatically promoted the accumulation of lobetyolin and total saponins in the hairy roots (Fig. 8). Among 16 AtSPLs in Arabidopsis, AtSPL 9 is the only one that has been reported to regulate biosynthesis of secondary metabolites [24, 25]. AtSPL9 negatively regulates anthocyanin accumulation by preventing the formation of MBW complex [24], and it positively regulates the formation of (E)- $\beta$-caryophyllene by binding to the promoter of sesquiterpene synthase gene TPS21 and activates its expression [25]. Our results indicated that CPSPL2 and CpSPL 10 are potential candidates for genetic improvement of $C$. pilosula because they can significantly promote not only the growth of hairy roots, but also accumulation of lobetyolin and total saponins. The molecular mechanism that CPSPL2 and CpSPL10 function in hairy roots needs to be addressed in the future.

\section{Conclusions}

In this study, we identified 15 CPSPL genes, which were supported by confirmation of the SBP domain, based on the genome data of $C$. pilosula. Ten of 15 CpSPLs were predicted to be directly targeted by miR156, including CpSPL3-6, CpSPL9-13, and CPSPL15. All CPSPLs were clustered into seven groups and members in the same group share similar gene structure and conserved motif composition. The spatiotemporal expression analysis of $15 \mathrm{CPSPLs}$ showed that CpSPL gene family had

Page $8 / 20$ 
various expression patterns. The expression levels of most $C p S P L s$ significantly changed under $\mathrm{NaCl}, \mathrm{MeJA}$, or $\mathrm{ABA}$ treatment, and CPSPL5 and CPSPL8 showed higher change folds under different treatments. Overexpression of CpSPL2 or CPSPL 10 significantly promoted not only the growth of hairy roots, but also the accumulation of lobetyolin and total saponins. $C P S P L 2$ and $C P S P L 10$ are potential candidates for genetic improvement of $C$. pilosula. These results established a foundation for further investigation of $C P S P L s$ and provided novel insights into their biological functions.

\section{Abbreviations}

Not applicable.

\section{Declarations}

Ethics approval and consent to participate

Not applicable.

Consent for publication

Not applicable.

\section{Availability of data and materials}

All data generated or analysed during this study are included in this published article and its supplementary information files.

\section{Competing Interests}

There is no conflict of interest among authors.

\section{Funding}

This work was supported by the Major Project of Shaanxi Province, China (Grant No.2017ZDXM-SF-005) and The Youth Innovation Team of Shaanxi Universities. The funders did not design the experiment or draft and revise the manuscript.

\section{Authors' contributions}

JY performed the experiments, analyzed the data, and wrote the draft manuscript. ZG prepared the materials and assisted in the experiments. YC, RC and WW assisted in manuscript preparation and helped modify some Figs. $X Y$ and $X C$ conceived and coordinated the overall study and revised the manuscript. All authors read and approved the final version of the manuscript.

\section{Acknowledgements}

Not applicable.

\section{References}

1. Luscombe NM, Austin SE, Bermanand HM, Thornton JM. An overview of the structures of protein-DNA complexes. Genome Biol. 2000;1(1):REVIEWS001.

2. Jin J, Tian F, Yang DC, Meng YQ, Kong L, Luo J, Gao G. PlantTFDB 4.0: toward a central hub for transcription factors and regulatory interactions in plants. Nucleic Acids Res. 2017;45(D1):D1040-D1045.

3. Klein J, Saedler H, Huijser P. A new family of DNA binding proteins includes putative transcriptional regulators of the Antirrhinum majus floral meristem identity gene SQUAMOSA. Mol Gen Genet. 1996;250(1):7-16.

4. Yamasaki K, Kigawa T, Inoue M, Tateno M, Yamasaki T, Yabuki T, Aoki M, Seki E, Matsuda T, Nunokawa E, Ishizuka Y, Terada T, Shirouzu M, Osanai T, Tanaka A, Seki M, Shinozaki K, Yokoyama S. A novel zinc-binding motif revealed by solution 
structures of DNA-binding domains of Arabidopsis SBP-family transcription factors. J Mol Biol., 2004;337(1):49-63.

5. Birkenbihl RP, Jach G, Saedler H, Huijser P. Functional dissection of the plant-specific SBP-domain: overlap of the DNAbinding and nuclear localization domains. J Mol Bio. 2005;352(3):585-596.

6. Yang Z, Wang X, Gu S, Hu Z, Xu H, Xu C. Comparative study of SBP-box gene family in Arabidopsis and rice. Gene. 2008;407(1):1-11.

7. Preston JC, Hileman LC. Functional evolution in the plant SQUAMOSAPROMOTER BINDING PROTEIN-LIKE (SPL) gene family. Front Plant Sci. 2013; 4:80.

8. Xu M, Hu T, Zhao J, Park MY, Earley KW, Wu G, Yang L, Poethig RS. Developmental functions of miR156-regulated SQUAMOSA PROMOTER BINDING PROTEIN-LIKE (SPL) genes in Arabidopsis thaliana. PLoS Genet. 2016;2(8):e1006263.

9. Xie K, Wu C, Xiong L. Genomic organization, differential expression, and interaction of SQUAMOSA promoter-binding-like transcription factors and microRNA156 in rice. Plant Physiol, 2006;142:280-293.

10. Tripathi RK, Goel R, Kumari S, Dahuja A. Genomic organization, phylogenetic comparison, and expression profiles of the SPL family genes and their regulation in soybean. Dev Genes Evol. 2017;227(2):101-119.

11. Salinas M, Xing S, Höhmann S, Berndtgen R, Huijser P. Genomic organization, phylogenetic comparison and differential expression of the SBP-box family of transcription factors in tomato. Planta. 2012;235(6):1171-1184.

12. Li J, Hou H, Li X, Xiang J, Yin X, Gao H, Zheng Y, Bassett CL, Wang X. Genome-wide identification and analysis of the SBPbox family genes in apple (Malus $\times$ domestica). Plant Physiol Biochem. 2013;70:100-114.

13. Zhang L, Wu B, Zhao D, Li C, Shao F, Lu S. Genome wide analysis and molecular dissection of the SPL gene family in Salvia miltiorrhiza. J Integr Plant Biol. 2014;56:38-50.

14. Hou H, Li J, Gao M, Singer SD, Wang H, Mao L, Fei Z, Wang X. Genomic organization, phylogenetic comparison and differential expression of the SBP-Box family genes in grape. PLoS ONE. 2013; 8: e59358.

15. Pan F, Wang Y, Liu H, Wu M, Chu W, Chen D, Xiang Y. Genome-wide identification and expression analysis of SBP-like transcription factor genes in Moso bamboo (Phyllostachys edulis). BMC Genomics. 2017;18(1):486.

16. Zhang HX, Jin JH, He YM, Lu BY, Li DW, Chai WG, Khan A, Gong ZH. Genome-wide identification and analysis of the SBPbox family genes under Phytophthora capsici stress in pepper (Capsicum annuum). Front Plant Sci. 2016;7:504.

17. Zhang SD, Ling LZ. Genome-wide identification and evolutionary analysis of the SBP-box gene family in castor bean. PLoS ONE. 2014;9(1):e86688.

18. Gandikota M, Birkenbihl RP, Höhmann S, Cardon GH, Saedler H, Huijser P. The miRNA156/157 recognition element in the 3' UTR of the Arabidopsis SBP box gene SPL3 prevents early flowering by translational inhibition in seedlings. Plant J. 2010;49(4):683-693.

19. Lal S, Pacis LB, Smith HM. Regulation of the SQUAMOSA PROMOTER-BINDING PROTEIN-LIKE genes/microRNA156 module by the homeodomain proteins PENNYWISE and POUND-FOOLISH in Arabidopsis. Mol Plant. 2011;4:1123-1132.

20. Hyun Y, Richter R, Vincent C, Martinez-Gallegos R, Porri A, Coupland G. Multi-layered regulation of SPL 15 and cooperation with SOC1 integrate endogenous flowering pathways at the Arabidopsis shoot meristem. Dev Cell. 2015;37(3):254-266.

21. Gao R, Wang Y, Gruber MY, Hannoufa A. miR156/SPL10 modulates lateral root development, branching and leaf morphology in Arabidopsis by silencing AGAMOUS-LIKE 79. Front Plant Sci. 2017;8:2226.

22. Ye BB, Zhang K, Wang JW. The role of miR156 in rejuvenation in Arabidopsis thaliana. J Integr Plant Biol. 2019;62(5):550555.

23. Barrera-Rojas CH, Rocha GH, Polverari L, Pinheiro Brito DA, Batista DS, Notini MM, Cruz AC, Morea EG, Sabatini S, Otoni WC, Nogueira FT. miR156-targeted SPL10 controls root meristem activity and root-derived de novo shoot regeneration via cytokinin responses. J Exp Bot. 2020;71(3):934-950.

24. Gou JY, Felippes FF, Liu CJ, Weigel D, Wang JW. Negative regulation of anthocyanin biosynthesis in Arabidopsis by a miR156-targeted SPL transcription factor. Plant Cell. 2011;23(4):1512-1522.

25. Yu ZX, Wang LJ, Zhao B, Shan CM, Zhang YH, Chen DF, Chen XY. Progressive regulation of sesquiterpene biosynthesis in Arabidopsis and Patchouli (Pogostemon cablin) by the miR156-targeted SPL transcription factors. Mol Plant. 2015;8:98-

Page $10 / 20$ 
110.

26. Feyissa BA, Arshad M, Gruber MY, Kohalmi SE, Hannoufa A. The interplay between miR156/SPL13 and DFR/WD40-1 regulate drought tolerance in alfalfa. BMC Plant Biol. 2019;19:434.

27. Mermod M, Takusagawa M, Kurata T, Kamiya T, Fujiwara T, Shikanai T. SQUAMOSA promoter-binding protein-like 7 mediates copper deficiency response in the presence of high nitrogen in Arabidopsis thaliana. Plant Cell Rep. 2019;38: $835-$

28. Miura K, Ikeda M, Matsubara A, Song XJ, Ito M, Asano K, Matsuoka M, Kitano H, Ashikari M. OsSPL 14 promotes panicle branching and higher grain productivity in rice. Genet. 2010;42:545-549.

29. Wang SK, Wu K, Yuan QB, Liu XY, Liu ZB, Lin XY, Zeng RZ, Zhu HT, Dong GJ, Qian Q, Zhang G, Fu X. Control of grain size, shape and quality by OSSPL 16 in rice. Genet. 2012;44:950-954.

30. Xiong J, Bai Y, Ma C, Zhu H, Zheng D, Cheng Z. Molecular cloning and characterization of SQUAMOSA-promoter binding protein-like gene FVSPL 10 from Woodland Strawberry (Fragaria vesca). Plants (Basel). 2019;8(9):342.

31. Committee for the Pharmacopoeia of the People's Republic of China, Pharmacopoeia of the People's Republic of China, Part I. China Medical Science Press: Beijing; 2020;293-294.

32. Li FJ, Wang ZC, Yang K. Summary of recent research on Codonopsis pilosula. Technol Inf. 2008;35:422-440.

33. Li D, Li ZL. The research status of that Codonopsis pilosula polysaccharide is as an immune adjuvant. Guide of China Medicine. 2013;11:56-57.

34. He JY, Ma N, Zhu S, Komatsu K, Li ZY, Fu WM. The genus Codonopsis (Campanulaceae): a review of phytochemistry, bioactivity and quality control. J Nat Med. 2015;69(1):1-

35. Yoon IS, Cho SS. Effects of lobetyolin on xanthine oxidase activity in vitro and in vivo: weak and mixed inhibition. Nat Prod Res. 2019;29:1-

36. He W, Tao W, Zhang F, Jie Q, He Y, Zhu W, Tan J, Shen W, Li L, Yang Y, Cheng H, Sun D. Lobetyolin induces apoptosis of colon cancer cells by inhibiting glutamine metabolism. J Cell Mol Med. 2020;24(6):3359-

37. Gao JP, Wang D, Cao LY, Sun HF. Transcriptome sequencing of Codonopsis pilosula and identification of candidate genes involved in polysaccharide biosynthesis. PLoS ONE. 2015;10:e0117342.

38. Ji JJ, Feng Q, Sun HF, Zhang XJ, Li XX, Li JK, Gao JP. Response of bioactive metabolite and biosynthesis related genes to methyl jasmonate elicitation in Codonopsis pilosula. Molecules. 2019;24:533.

39. Yang J, Yang X, Li B, Lu X, Kang J, Cao X. Establishment of in vitro culture system of Codonopsis pilosula transgenic hairy root. 3 Biotech. 2020;10:137.

40. Yang J, Yang XZ, Kuang Z, Li B, Lu XY, Cao XY, Kang JF. Selection of suitable reference genes for qRT-PCR expression analysis of Codonopsis pilosula under different experimental conditions. Molecular biology reports. 2020;47(6): 4169-

41. Livak KJ, Schmittgen TD. Analysis of relative gene expression data using real-time quantitative PCR and the $2^{-\triangle \triangle C T}$ Methods. 2001;25:402-408.

42. Axtell MJ, Bowman JL. Evolution of plant microRNAs and their targets. Trends Plant Sci. 2008;13:343-349.

43. Ye BB, Shang GD, Pan Y, Xu ZG, Zhou CM, Mao YB, Bao N, Sun L, Xu T, Wang JW. AP2/ERF transcription factors integrate age and wound signals for root regeneration. Plant Cell. 2020;32:226-

44. Jung JH, Lee HJ, Ryu JY, Park CM. SPL3/4/5 integrate developmental aging and photoperiodic signals into the FT-FD module in Arabidopsis Mol Plant. 2016;9(12):1647-1659.

45. Zhou Q, Zhang S, Chen F, Liu B, Wu L, Li F, Zhang J, Bao M, Liu G. Genome-wide identification and characterization of the SBP-box gene family in Petunia. BMC Genomics. 2018;19(2):193-211.

46. Li C, Lu S. Molecular characterization of the SPL gene family in Populus trichocarpa. BMC Plant Biol. 2014;14:131.

47. Zeng RF, Zhou JJ, Liu SR, Gan ZM, Zhang JZ, Hu CG. Genome-wide identification and characterization of SQUAMOSApromoter-binding protein (SBP) genes involved in the flowering development of Citrus Clementina. Biomolecules. 2019;9(2):66. 
48. Chao LM, Liu YQ, Chen DY, Xue XY, Mao YB, Chen XY. Arabidopsis transcription factors SPL 1 and SPL 12 confer plant thermotolerance at reproductive stage. Mol Plant. 2017;10:735-

49. Matthews C, Arshad M, Hannoufa A. Alfalfa response to heat stress is modulated by microRNA156. Physiol Plant. 2019;165(4):830-

50. Gou J, Debnath S, Sun L, Flanagan A, Tang Y, Jiang Q, Wen J, Wang ZY. From model to crop: functional characterization of $S P L 8$ in truncatula led to genetic improvement of biomass yield and abiotic stress tolerance in alfalfa. Plant Biotechnol J. 2018;16(4):951-962.

\section{Appendix A. Supplemental Files}

The supplemental tables were not provided with this version of the manuscript.

Table S1. Primer sequences used in the study

Table S2. cDNA sequences of 15 SPLs in Codonopsis pilosula

Table S3. The conserved motifs of SPL in Codonopsis pilosula

Table S4. Putative cis-acting elements present in SPL promoters of Codonopsis pilosula

\section{Figures}


CPSPL3

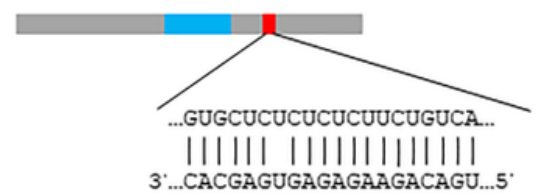

AtmiR156

CPSPL5

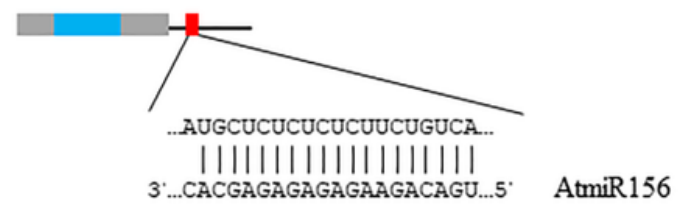

CPSPL9

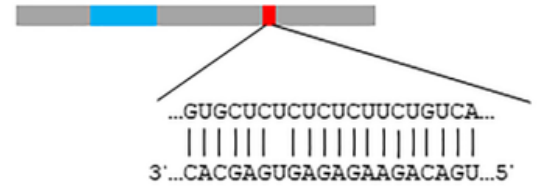

3....CACGAGUGAGAGAAGACAGU...

AtmiR156

\section{CPSPL11}

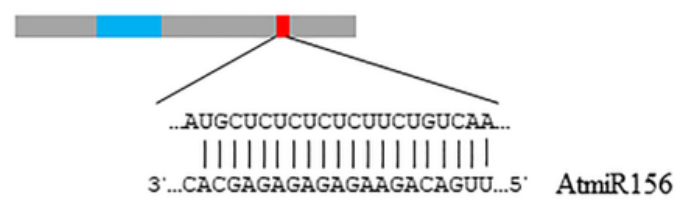

\section{CpSPL13}

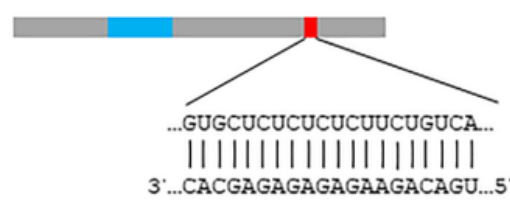

CPSPL4

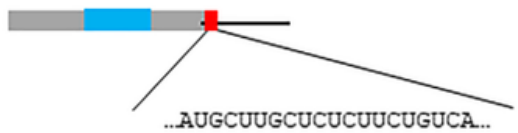

|l|| ||||||||||||

3....CACGAGUGAGAGAAGACAGU....5' AtmiR156

CPSPL6

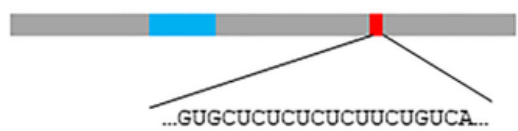

|l|||| ||||||||||||

3....CACGAGUGAGAGAAGACAGC...5' AtmiR156

CPSPL10

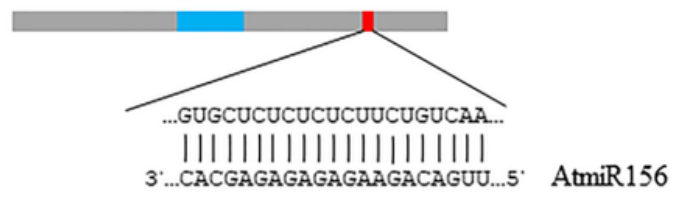

CpSPL12

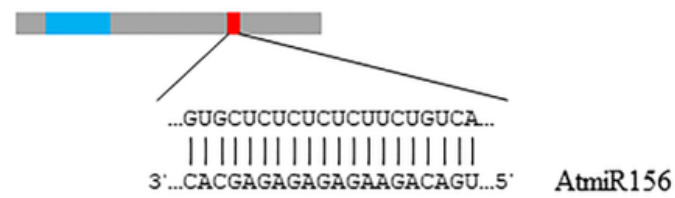

CPSPL15

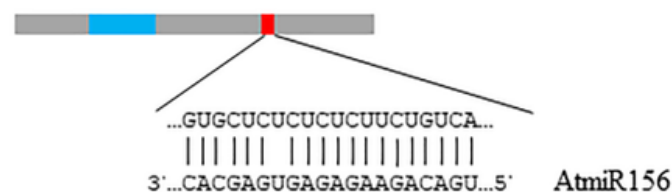

\section{Figure 1}

Binding sites of AtmiR156 in CpSPL genes. The CpSPLs regulated by miR156. The gray boxes represent the CDS of CpSPL genes. The blue boxes represent the conserved SBP domain. The red boxes represent the miR156 target site. 


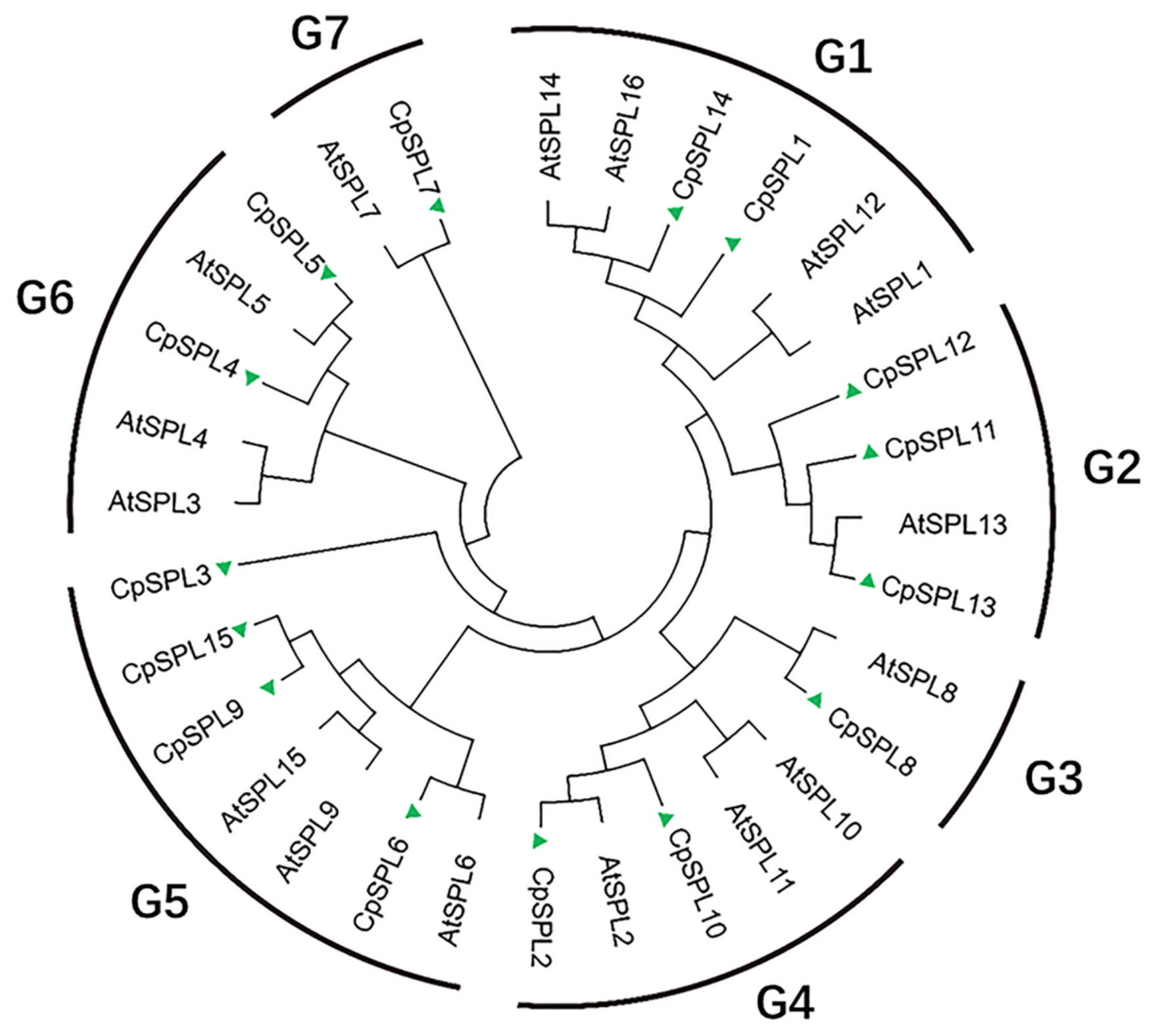

Figure 2

An ML phylogenetic tree of the SPLs from Codonopsis pilosula and Arabidopsis thaliana. 


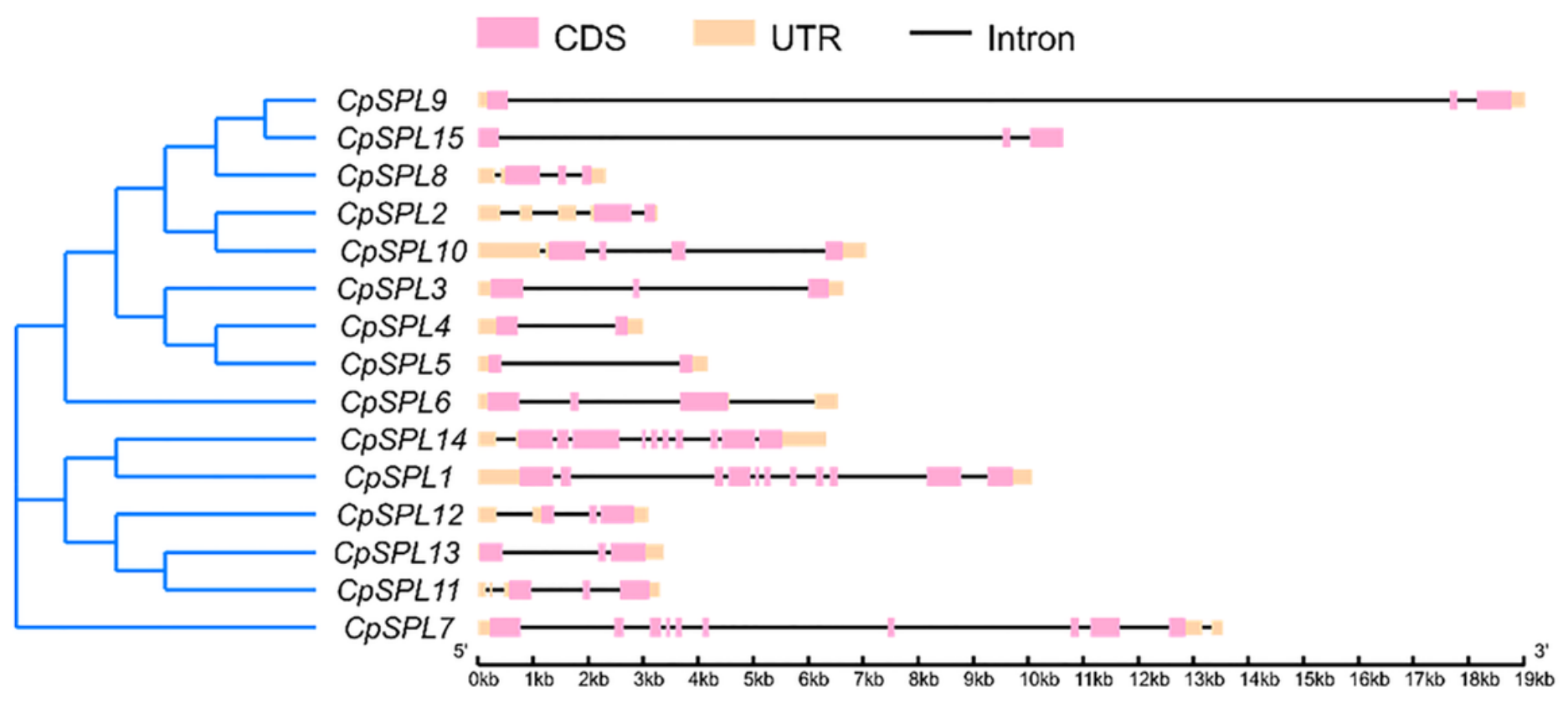

\section{Figure 3}

Exon-intron organization structures of $15 \mathrm{SPL}$ genes in Codonopsis pilosula. Exons are represented by pink rectangles, introns are represented by black lines, UTRs are represented by orange rectangles. 


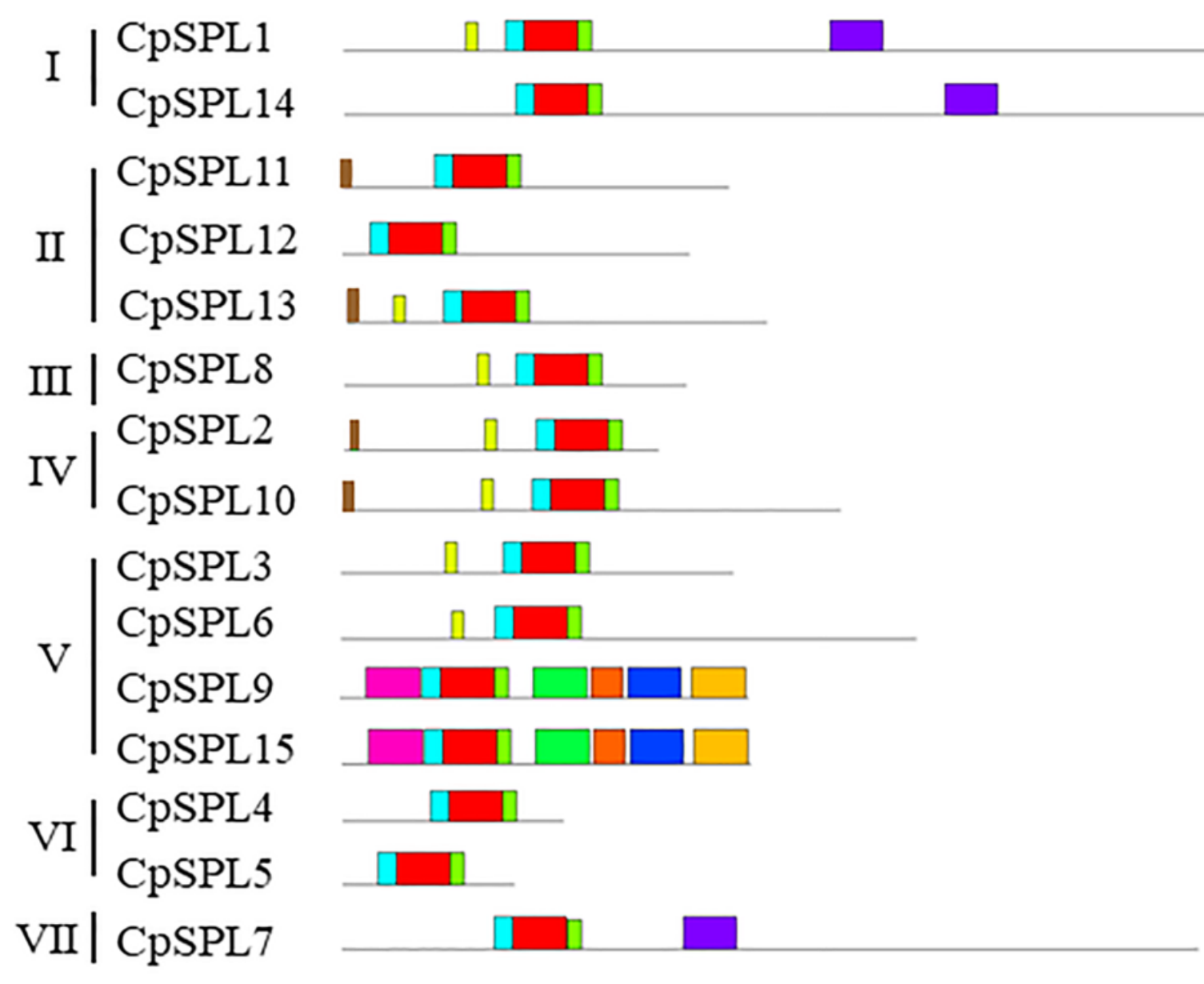

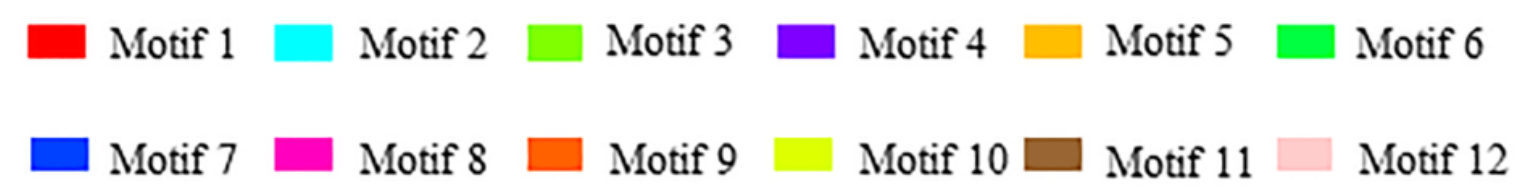

Figure 4

The analysis of conserved motifs of SPLs in Codonopsis pilosula. 

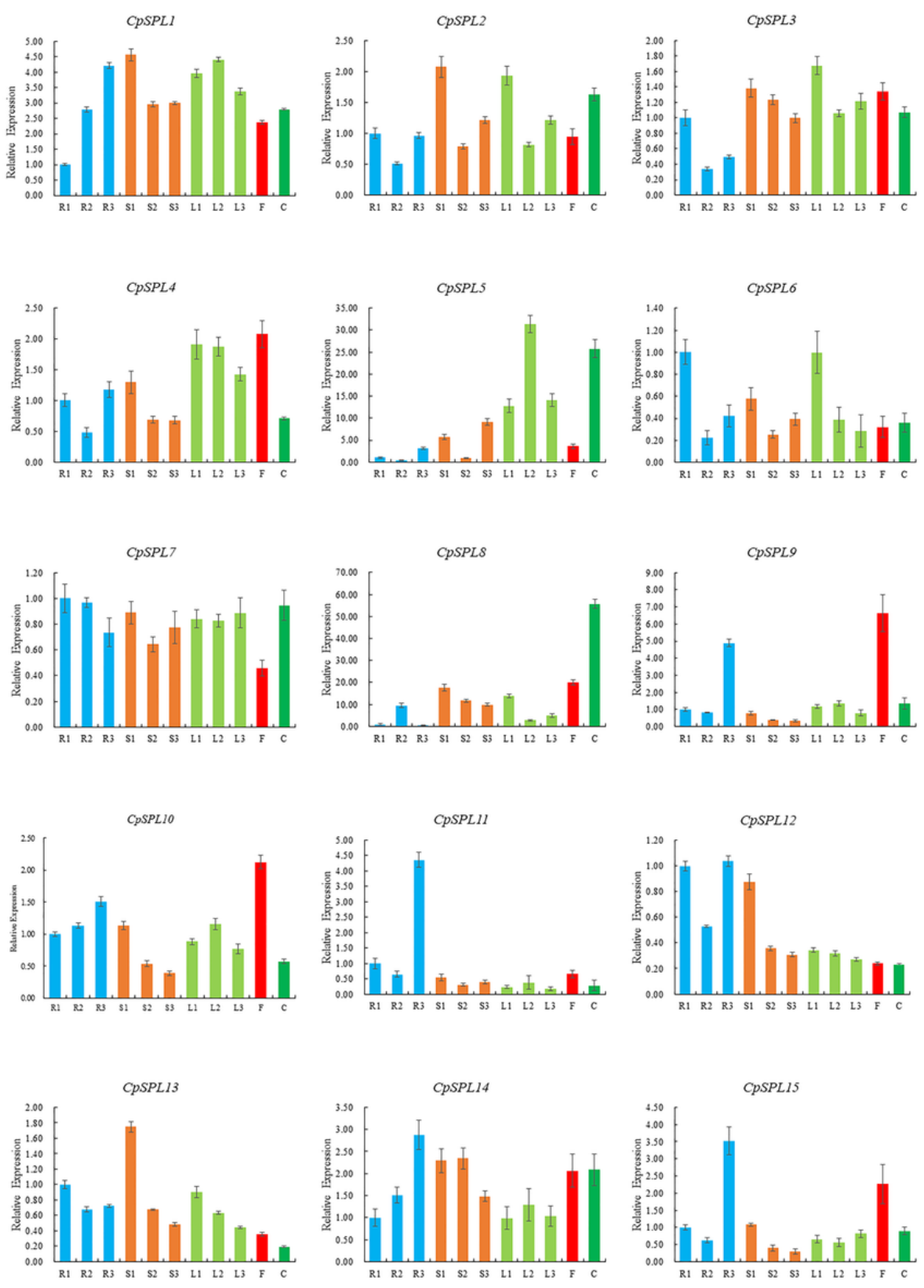

Figure 5

Spatiotemporal expression analysis of 15 SPL genes in Codonopsis pilosula. R1, R2, and R3 represent roots from one-, two-, and three-month-old seedlings, respectively; S1, S2, and S3 represent stems from one-, two-, and three-month-old seedlings, respectively; L1, L2, and L3 represent leaves from one-, two-, and three-month-old seedlings, respectively; F: flower; C: calyx. 

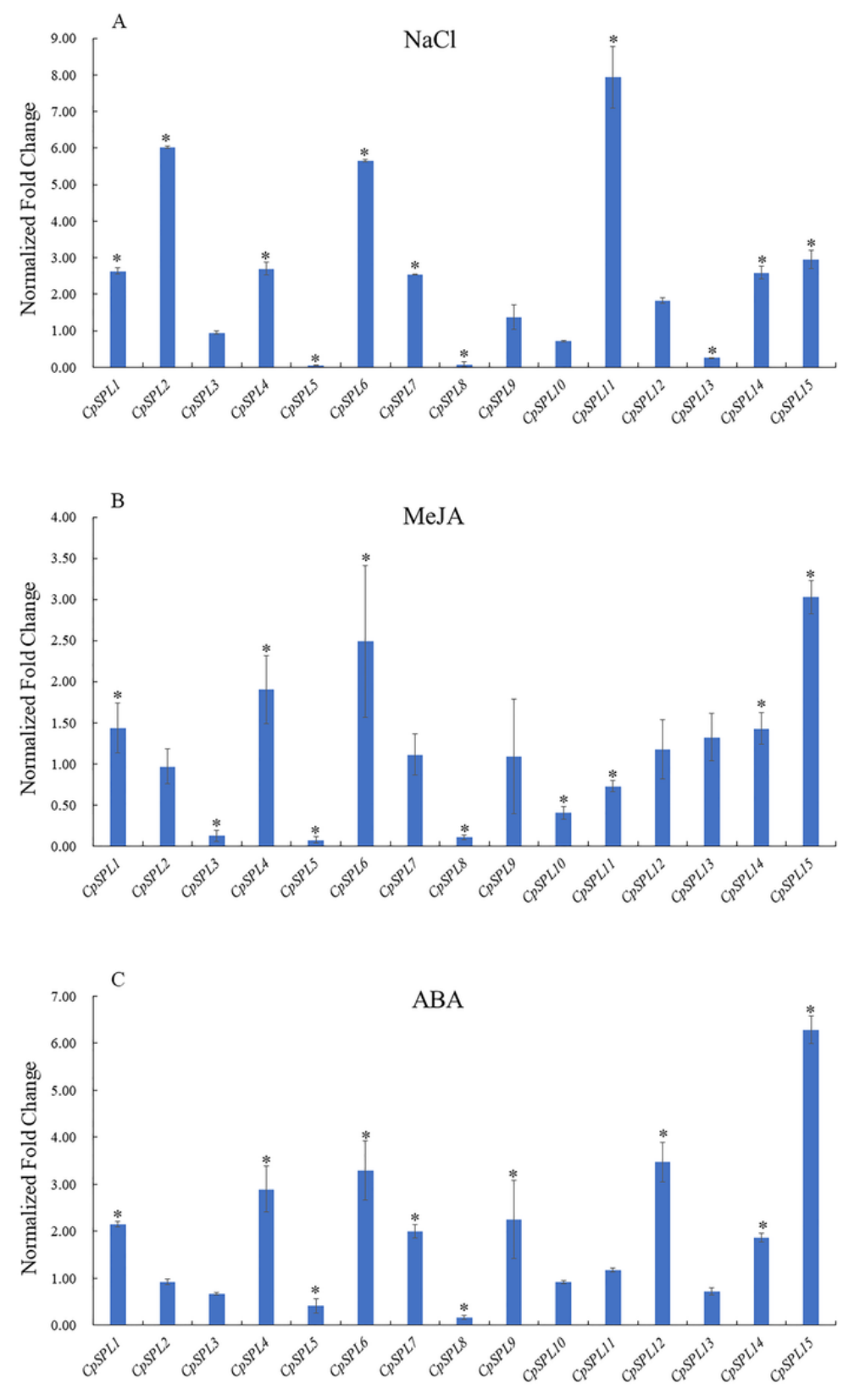

\section{Figure 6}

Relative expression levels of $15 \mathrm{SPL}$ genes in Codonopsis pilosula under $\mathrm{NaCl}$, methyl jasmonate (MeJA), and abscisic acid (ABA) treatment. Change multiples are relative to the expression level of each gene treated with ddH2O (set to 1). * means the value is significantly different from the control at $p<0.05$. 

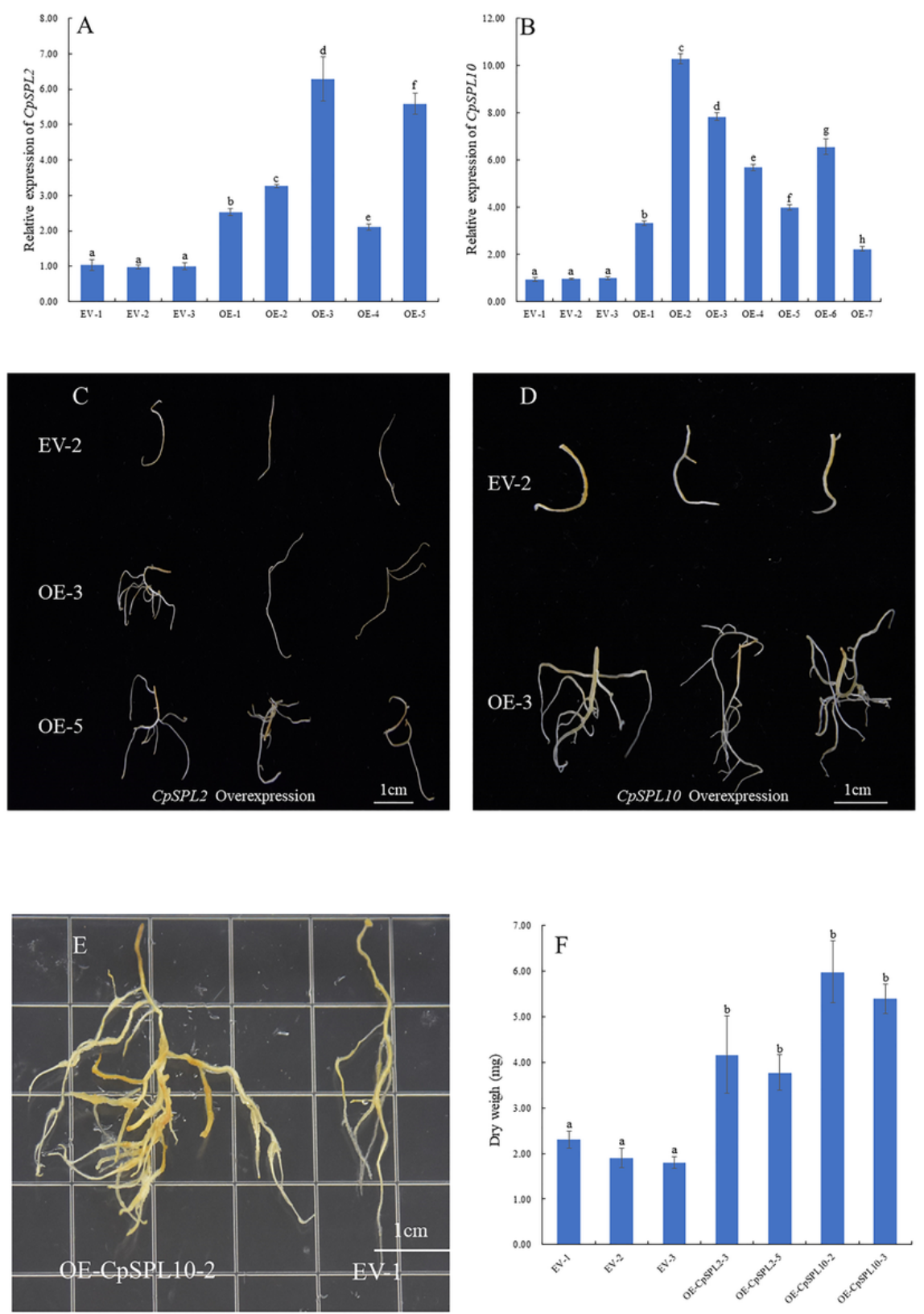

\section{Figure 7}

Identification and growth phenotype of CpSPL2 or CpSPL10-overexpressing (OE) Codonopsis pilosula transgenic hairy roots. (A) Expression levels of CpSPL2 in CpSPL2-OE lines. (B) Expression levels of CpSPL10 in CpSPL10-OE lines. (C) Growth phenotype of empty vector (EV) and CpSPL2-OE lines when $1 \mathrm{~cm}$ hairy roots were cultured for one month. (D) Growth phenotype of EV and CpSPL10-OE lines when $1 \mathrm{~cm}$ hairy roots were cultured for one month. (E) Growth phenotype of EV and CpSPL10-OE lines when $1 \mathrm{~cm}$ hairy roots were cultured for two months. (F) The biomass of EV, CpSPL2-OE and CpSPL10-OE lines when $1 \mathrm{~cm}$ hairy roots were cultured for one months. One-way ANOVA (followed by Tukey's comparisons) tested for significant differences among means (indicated by different letters at $p<0.05$ ). 

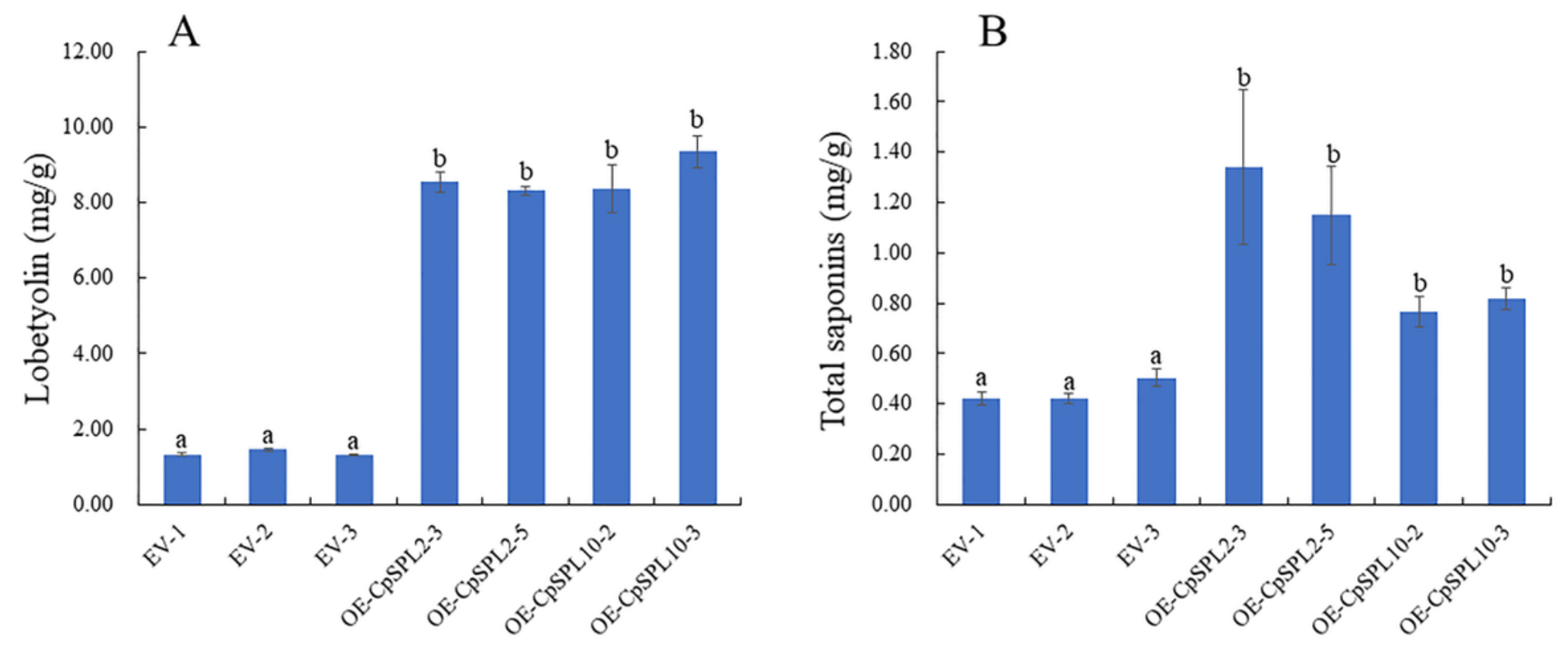

Figure 8

Concentration of lobetyolin and total saponins contents in Codonopsis pilosula transgenic hairy roots. One-way ANOVA (followed by Tukey's comparisons) tested for significant differences among means (indicated by different letters at $p<0.05$ ). 